\title{
Association between the Mediterranean lifestyle, metabolic syndrome and mortality: a whole-country cohort in Spain
}

\author{
Mercedes Sotos-Prieto 1,2,6* $\mathbb{0}$, Rosario Ortolá1, Miguel Ruiz-Canela ${ }^{3,4}$, Esther Garcia-Esquinas ${ }^{1}$, \\ David Martínez-Gómez ${ }^{1,6}$, Esther Lopez-Garcia 1,6, Miguel Ángel Martínez-González 3,4,5 \\ and Fernando Rodriguez-Artalejo $0^{1,6}$
}

\begin{abstract}
Background: Evidence is limited about the joint health effects of the Mediterranean lifestyle on cardiometabolic health and mortality. The aim of this study was to evaluate the association of the Mediterranean lifestyle with the frequency of the metabolic syndrome (MS) and the risk of all-cause and cardiovascular mortality in Spain.

Methods: Data were taken from ENRICA study, a prospective cohort of 11,090 individuals aged 18+ years, representative of the population of Spain, who were free of cardiovascular disease (CVD) and diabetes at 2008-2010 and were followed-up to 2017. The Mediterranean lifestyle was assessed at baseline with the 27-item MEDLIFE index (with higher score representing better adherence).

Results: Compared to participants in the lowest quartile of MEDLIFE, those in the highest quartile had a multivariable-adjusted odds ratio 0.73 (95\% confidence interval (CI) 0.5, 0.93) for MS, 0.63. (0.51, 0.80) for abdominal obesity, and $0.76(0.63,0.90)$ for low HDL-cholesterol. Similarly, a higher MELDIFE score was associated with lower HOMA-IR and highly-sensitivity C-reactive protein (P-trend $<0.001$ ). During a mean follow-up of 8.7 years, 330 total deaths (74 CVD deaths) were ascertained. When comparing those in highest vs. lowest quartile of MEDLIFE, the multivariable-adjusted hazard ratio $(95 \% \mathrm{Cl})$ was $0.58(0.37,0.90)$ for total mortality and $0.33(0.11,1.02)$ for cardiovascular mortality.
\end{abstract}

Conclusions: The Mediterranean lifestyle was associated with lower frequency of MS and reduced all-cause mortality in Spain. Future studies should determine if this also applies to other Mediterranean countries, and also improve cardiovascular health outside the Mediterranean basin.

Keywords: Mediterranean lifestyle, Cardiovascular risk factors, Metabolic syndrome mortality, Cohort

\section{Background}

The Mediterranean diet (MedDiet) is the culturally rooted diet traditionally consumed by the populations living in the Mediterranean basin [1]. Despite some

\footnotetext{
*Correspondence: mercedes.sotos@uam.es

${ }^{1}$ Department of Preventive Medicine and Public Health, School of Medicine, Universidad Autónoma de Madrid, IdiPaz (Instituto de Investigación Sanitaria Hospital Universitario La Paz), and CIBERESP (CIBER of Epidemiology and Public Health), Madrid, Spain

Full list of author information is available at the end of the article
}

between-country variations, the MedDiet in Spain reflects a food consumption pattern rich in fruits and vegetables, legumes, whole grains, olive oil, fish and nuts, with greater consumption of white or lean meats than of red or processed meats, moderate consumption of dairy products, and intake of small amounts of wine, mainly red wine and with meals. In addition, the MedDiet reflects other influences of the Mediterranean culture, including a rich social interaction, "siesta" (short nap after meals), eating with friends and family, traditional recipes and culinary practices, and a substantial amount 
of physical activity; all these elements characterize the Mediterranean lifestyle, as represented in the MedDiet pyramid elaborated though a consensus of nutrition experts and the MedDiet Foundation [2].

There is robust evidence, accumulated over the last decades, of the beneficial effects of the MedDiet on chronic diseases, including the metabolic syndrome (MS) [3-5], type 2 diabetes [6-9] and obesity [10,11] as well as cardiovascular disease [12-16], cancer [17] and all-cause mortality $[13,18-20]$, both in Mediterranean and nonMediterranean countries.

More recently, epidemiological studies have started to evaluate the joint health effect of multiple health behaviors and biological CVD risk factors combined into a single predefined score [21-27]. For example, Li et al., found that adopting four out of five low risk lifestyle factors (never smoking, healthy body mass index (BMI), moderate-to-vigorous physical activity, moderate alcohol intake, and a higher diet quality) was associated with about a 10-year increase in life expectancy [25]. However, there is a lack of evidence about the joint and synergistic health effects of the different components of the Mediterranean lifestyle. The Mediterranean lifestyle (MEDLIFE) index is a score of the set of behaviors that best characterize the adoption of a traditional Mediterranean lifestyle $[28,29]$. It was developed and validated in a Spanish working population $[28,29]$, and a higher score has been associated with lower frequency of CVD risk factors in 366 workers from Croatia [30], and reduced mortality in a Spanish cohort of university graduates. However, no previous research has evaluated the influence of the MEDLIFE index on cardiovascular health in the adult population of a whole Mediterranean country. Therefore, the aim of this study was to evaluate the association of the MEDLIFE index with the prevalence of the MS and other biological CVD risk factors, and with the risk of allcause and CVD death, on a representative sample of the adult population of Spain.

\section{Research design and methods Study population}

The Study on Nutrition and Cardiovascular Risk in Spain (ENRICA) is a prospective cohort study that consists of 13,105 individuals aged 18 years or older, who were recruited between June 2008 and October 2010 through random stratified cluster sampling to ensure that they were a representative sample of the non-institutionalized adult population of Spain. First, the sample was stratified by province and size of municipality. Second, clusters were selected randomly in two stages: municipalities and census sections. Finally, the households within each section were selected proportionally to the sex and age distribution of the Spanish population. Detailed information about the methods and sample collection process have been published elsewhere [31]. Trained and certified staff collected information in three stages: a phone interview and two subsequent home visits. The phone interview obtained data on sociodemographic factors, health behaviors, self-rated health and the existence of a previous medical diagnosis of a set of chronic conditions. In the first home visit, blood and urine samples were collected and sent to a central laboratory for analytical determinations; and in the second visit, a validated electronic diet history was obtained and a physical examination was performed [32]. Study participants have been followed-up to December of 2017 (mean follow-up to 8.7 years, SD 0.87) to assess their vital status and the cause of death.

Of the 13,105 participants at baseline, 921 were excluded because of missing information on diet or implausible reported energy intake $(<800 \mathrm{kcal}$ or $>5000 \mathrm{kcal}$ for men, and $<500 \mathrm{kcal}$ or $>4000 \mathrm{kcal}$ for women). We also excluded those lacking data on their clinical CVD risk factors $(n=115)$ and those who had a previous medical diagnosis of CVD (heart failure, myocardial infarction or stroke $(n=262)$ or diabetes at baseline $(n=716)$. That left a sample size of 11,091 for the analyses (Additional file 1: Figure S1).

Study participants gave written informed consent, and the study was approved by the Clinical Research Ethics Committee of La Paz University Hospital in Madrid (Trial registration: NCT02804672).

\section{Study variables \\ Mediterranean lifestyle index}

Baseline information on habitual food consumption in the preceding year was obtained with a validated computerized face-to-face diet history (DH-ENRICA), developed from that used in the European Prospective Investigation into Cancer and Nutrition (EPIC)-cohort study in Spain [32]. The diet history included 880 different foods and a set of 127 photographs that helped to quantify food portions. Standard food composition tables were used to estimate nutrient and energy intake [32].

Physical activity was ascertained using the validated European Prospective EPIC-cohort questionnaire [33]. Leisure-time activity was reported as the time spent in walking, cycling and other types of exercise, as well as in household chores, gardening and do-it-yourself activities. Time spent in sedentary behaviors, including while watching $\mathrm{TV}$, at the computer use, reading, commuting, and listening to music was also obtained using the Nurses' Health Study questionnaire validated in Spain [34]. Data regarding sleep, nap, conviviality and habits around meals were also self-reported.

Based on the above information, the MEDLIFE index was calculated following the criteria published by 
Sotos-Prieto et al. [28, 29]. Briefly, the MEDLIFE consists of 28 items divided into three blocks: (1) food consumption (15 items); (2) dietary habits (7 items); and (3) physical activity, rest and conviviality (6 items). However, we could not compute the item on water consumption for not having appropriate information in the ENRICA study. Each item of the score weights 0 (negative) or 1 point (positive), and the score ranged from 0 to 27 (best adherence) (Additional file 1: Table S1).

\section{Outcome measurements}

Clinical CVD risk factors According to the harmonized definition [35], MS was defined as having at least three of the following five criteria: abdominal obesity (waist circumference $\geq 102 \mathrm{~cm}$ in men, or $\geq 88 \mathrm{~cm}$ in women); elevated blood glucose $(\geq 100 \mathrm{mg} / \mathrm{dl}$ or treatment with antidiabetic drugs); high blood pressure (systolic $\geq 130 \mathrm{mmHg}$ or diastolic $\geq 85 \mathrm{mmHg}$, or receiving antihypertensive drugs); triglycerides $\geq 150 \mathrm{mg} / \mathrm{dl}$; and serum high-density lipoprotein (HDL) cholesterol $<40 \mathrm{mg} / \mathrm{dl}$ in men or $<50 \mathrm{mg} / \mathrm{dl}$ in women. Waist circumference was measured with a flexible non-stretchable tape at the midpoint between the last rib and the iliac crest after a normal expiration. Blood glucose was determined by the glucose oxidase technique after $12 \mathrm{~h}$ fasting. Blood pressure was measured by trained personnel using a standard protocol, with validated automatic devices (Omron M6) and cuffs of 3 sizes according to arm circumference. Two sets of blood pressure readings were taken separated by $90 \mathrm{~min}$. In each set, blood pressure was measured 3 times at 1-2-min intervals, after resting between 3 and 5 min in a seated position. For the analyses, average systolic and diastolic blood pressure was calculated for those who had at least 3 measurements, after discarding the first reading. HDL-cholesterol was measured with the direct method, by elimination/catalase. Triglycerides were determined by the glycerol phosphate oxidase method [36].

In addition, insulin was determined by immunoradiometric assay. The Homoeostatic Model Assessment for IR Index (HOMA-IR) was calculated by multiplying glucose in $\mathrm{mg} / \mathrm{dl}$ by insulin in $\mathrm{mU} / \mathrm{l}$ and dividing by 405 [37]. Finally, high-sensitivity C-reactive protein (hs-CRP) was assessed by latex-enhanced nephelometry to assess chronic inflammation.

Assessment of CVD and all-cause mortality The date and cause of death were ascertained from a computerized search of the vital registry of the Spanish National Institute of Statistics from baseline (2008-2010) to the end of follow-up on December 31, 2017; there is evidence of the completeness, accuracy, and reliability of this vital-status information [38-40]. The date and cause of death was determined from the death certificate by a nosologist and was coded according to the International Classification of Diseases, 10th Revision (ICD-10); specifically, CVD death was encoded as group I00-I99). Censoring was set at the date of death or at the end of the follow-up (December 31, 2017), whichever occurred first.

\section{Other variables}

At baseline, we collected information on sociodemographic and lifestyle characteristics including age, sex, educational level (no formal or primary education, secondary education, and university) and tobacco smoking (former, never, current) [31]. Also, weight and height were measured twice in each subject under standardized conditions; the BMI was calculated as weight (in $\mathrm{kg}$ ) divided by squared height (in $\mathrm{m}$ ) [31]. Finally, study participants reported their main drug treatments and the presence of the following physician-diagnosed diseases: chronic respiratory disease, coronary heart disease, stroke, heart failure, osteoarthritis, cancer, urinary infection, intestinal polyps, stomach ulcers, cirrhosis, Alzheimer's disease, Parkinson's disease, and depression requiring treatment.

\section{Statistical analysis}

The association between quartiles of MEDLIFE index and MS, and its individual components, at baseline was summarized with odds ratios (ORs) and their 95\% confidence interval (CI), obtained from multivariable logistic regression. In addition, multivariable-adjusted linear regression analyses were built to assess the association between MEDLIFE and HOMA-IR and hs-CRP, with results being summarized with $\beta$ and $95 \% \mathrm{CI}$, where $\beta$ coefficients represent adjusted differences of means between each of the upper quartiles of MEDLIFE and the lower quartile. $\mathrm{P}$ values for linear trend were calculated using quartiles of MEDLIFE as continuous variable. Three multivariable models were built based on the existing evidence that the variables are risk factors for the outcome [41] and observed association with MEDLIFE. Model 1 adjusted for sex, age, and education. Model 2 further adjusted for tobacco smoking (never, former, current), total energy intake (kcal/day), and BMI. And model 3 further adjusted for cancer, respiratory disease, depression, number of morbidities (excluding the previously mentioned conditions), and number of drug treatments. Natural splines adjusted for all covariates as in Model 3 were used to explore the dose-response association between MEDLIFE and biological CVD risk factors as continuous variables (waist circumference, blood glucose, systolic and diastolic blood pressure, triglycerides, HDL-cholesterol, HOMA-IR and hs-CRP).

Cox proportional regression models were fitted to assess the association between quartiles of adherence 
to the MEDLIFE score at baseline and all-cause mortality and CVD mortality, using the lowest quartile (Q1) as reference. $P$ values for linear trend were calculated using quartiles of MEDLIFE as continuous variable. In addition, hazard ratios (HRs) and 95\% CI were calculated for each 2-point increment in the MEDLIFE index. As above, we fitted three hierarchical models and we added a fourth one, which was further adjusted for some intermediate clinical risk factors (components of the MS). The proportional-hazards assumption was tested using the Schoenfeld residuals method. Multivariable-adjusted Kaplan-Meier graphs for survival were also elaborated to represent graphically the association between MEDLIFE and all-cause and CVD mortality.

In ancillary analysis, we stratified by morbidity (presence or not of any chronic condition), sex, age at baseline (or age at death only for the mortality analysis) and BMI. Likelihood ratio tests (with 3 degrees of freedom, the 3 upper quartiles of MEDLIFE and a dichotomous potential effect modifier) were performed to evaluate possible interactions. Other sensitivity analysis included the assessment of the association between each component block of the MEDLIFE and the clinical risk factors and mortality, using restricted cubic spline analysis with 3 knots for all-cause mortality or CVD mortality. Additionally, residual confounding by unmeasured confounders were addressed by calculating the E-value following the methodology described by VaderWeele and Ding [42].

The analyses were performed using Stata version 16.0 (Stata-Corp LLC, College Station, Texas). The survey command was used in the analyses to account for the complex sampling design. All p-values are two-sided and were considered statistically significant at $\mathrm{p}<0.05$.

\section{Results}

The actual MEDLIFE score ranged between 5 and 23 points in the cohort participants. Their baseline characteristics according to quartiles of the MEDLIFE index are shown in Table 1. Compared to those in the lowest quartile, participants with higher adherence to the MEDLIFE score had lower BMI, number of drug treatments, and morbidities. The frequency of the components of the MS was also lower. Additionally, participants with higher adherence were more likely to have higher education and to be never smokers, and less often had depression or respiratory disease.

A total of 1612 prevalent MS cases were documented. Table 2 shows the OR and 95\% CI of the MS, its individual components, and the average values of HOMAIR and hs-CRP according to quartiles of the MEDLIFE. After multivariable adjustment (model 3) and compared to participants in the lowest quartile of MEDLIFE, the OR for those in the highest quartile was $0.73(0.58,0.93)$ for MS, $0.63(0.51,0.80)$ for abdominal obesity, and 0.76 $(0.63,0.90)$ for low HDL-cholesterol. In addition, after adjustment for model 2 the inverse association was also statistically significant for elevated triglycerides and high blood pressure (P-trend 0.046). Similarly, higher adherence to the MELDIFE index was associated with lower levels of HOMA-IR and Hs-CRP (P-trend $<0.001$ ). The spline models showed a clear inverse dose-response relationship between the MEDLIFE index and waist circumference, fasting blood glucose, diastolic and systolic blood pressure, triglycerides, HOMA-IR, and Hs-CRP; by contrast, MEDLIFE was directly associated with HDLcholesterol (Fig. 1).

During a mean follow-up of 8.7 years (SD 0.87), 330 total deaths (including 74 CVD deaths) were ascertained. The cumulative incidence of all-cause mortality is $2 \%$ and $0.7 \%$ for CVD mortality over a mean followup of 8.7 years. The incidence rate of overall mortality is $0.93 / 100,000$ person-year $(95 \%$ CI $0.84,1.04)$; and for CVD deaths is $0.21 / 100,000$ person-year (95\% CI 0.17 , 0.26). Table 3 shows the multivariable-adjusted HR of all-cause and CVD mortality according to the MEDLIFE index. Compared to participants in the lowest quartile of MEDLIFE score, those in the highest quartile had lower risk of all-cause death [HR 0.58 (95\% CI 0.37, 0.90)] and of CVD death $[0.31(0.10,0.94)$ (model 3)]. Including the clinical CVD risk factors in the model did not materially change the results for total mortality but turned them into non-significant for CVD death (model 4). Each successive 2-point increase in the MEDLIFE score was significantly associated with a $11-12 \%$ reduced relative risk of all-cause death, with a significant linear trend in the models with the highest degree of adjustment. Multivariable-adjusted Kaplan-Meier graphs showed an inverse gradient in all-cause cause and CVD deaths over time according to quartiles of the MEDLIFE index (Fig. 2). Adjusted-restricted cubic splines showed a decrease for both all-cause and CVD mortality ( $p$ for linearity $=0.059$ for all-cause death and $\mathrm{p}=0.196$ for CVD death) (Additional file 1: Figure S2).

Results of secondary analyses did not show substantial changes in comparison with the main analysis. In stratified analyses, the association between MEDLIFE and MS did not vary by morbidity, sex, age at baseline, or BMI (All P for interaction >0.05) (Additional file 1: Table S2). Again, regarding all-cause mortality, no statistically significant interactions were found (Additional file 1: Table S3).

The associations between the individual block components of the MEDLIFE index and MS and mortality were primarily non-significant, except for the Block 3 (physical activity, rest, social habits, and conviviality); 
Table 1 Baseline characteristics of the ENRICA cohort according to quartiles of the MEDLIFE index

\begin{tabular}{|c|c|c|c|c|c|}
\hline & \multicolumn{4}{|c|}{ Mediterranean lifestyle index (MEDLIFE) } & \multirow[t]{2}{*}{$p$-value* } \\
\hline & Q1 (5-11 p) & Q2 (12-13p) & Q3 (14-15 p) & Q4 (16-23 p) & \\
\hline Number of participants & 3042 & 3435 & 2917 & 1696 & \\
\hline MEDLIFE score, range $0-27 p$ & $10.0(1.16)$ & $12.5(0.50)$ & $14.5(0.50)$ & $16.8(1.09)$ & $<0.001$ \\
\hline Block 1: Mediterranean food consumption, 0-15 p & $3.8(1.25)$ & $5.1(1.24)$ & $6.2(1.23)$ & $7.6(1.38)$ & $<0.001$ \\
\hline Block 2: Mediterranean eating habits, 0-6 p & $3.6(0.87)$ & $3.9(0.82))$ & $4.2(0.80)$ & $4.5(0.77)$ & $<0.001$ \\
\hline $\begin{array}{l}\text { Block 3: Mediterranean physical activity, rest, social } \\
\text { habits and conviviality, 0-6 p }\end{array}$ & $2.6(1.13)$ & $3.5(1.16)$ & $4.1(1.1)$ & $4.7(1.0)$ & $<0.001$ \\
\hline Sex, female, $\%$ & 54.9 & 53.0 & 52.5 & 52.4 & 0.189 \\
\hline Age, years & $47.8(17.0)$ & $45.8(16.1)$ & $45.9(16.0)$ & $46.3(15.0)$ & 0.311 \\
\hline Educational level, \% & & & & & $<0.001$ \\
\hline$\leq$ Primary & 30.3 & 27.2 & 24.7 & 22.6 & \\
\hline Secondary & 44.4 & 43.9 & 43.5 & 40.7 & \\
\hline University & 25.4 & 29.0 & 31.9 & 36.7 & \\
\hline Smoking status, \% & & & & & $<0.001$ \\
\hline Never & 44.2 & 47.7 & 50.3 & 48.5 & \\
\hline Former & 21.8 & 24.3 & 24.7 & 29.3 & \\
\hline Current & 34.0 & 28.0 & 25.0 & 22.2 & \\
\hline $\mathrm{BMI}, \mathrm{kg} / \mathrm{m}^{2}$ & $26.8(4.8)$ & $26.6(4.6)$ & $26.3(4.3)$ & $26.2(4.1)$ & $<0.001$ \\
\hline Energy intake, kcal/day & $2274(944)$ & $2232(864)$ & $2226(747)$ & $2238(683)$ & 0.08 \\
\hline Number of drug treatments & $0.71(1.32)$ & $0.65(1.26)$ & $0.61(1.22)$ & $0.62(1.21)$ & 0.006 \\
\hline Morbidities ${ }^{\mathrm{a}}, \%$ & 44.6 & 40.0 & 36.3 & 34.6 & $<0.001$ \\
\hline Number of morbidities & $0.66(1.0)$ & $0.63(0.95)$ & $0.56(0.90)$ & $0.52(0.88)$ & $<0.001$ \\
\hline \multicolumn{6}{|l|}{ Chronic conditions } \\
\hline Depression, \% & 7.9 & 7.2 & 5.4 & 4.2 & $<0.001$ \\
\hline Cancer, \% & 1.0 & 0.8 & 0.9 & 0.7 & 0.59 \\
\hline Respiratory disease, \% & 8.1 & 7.0 & 6.5 & 5.5 & 0.005 \\
\hline \multicolumn{6}{|l|}{ Components of the metabolic syndrome } \\
\hline Abdominal obesity ${ }^{b}$ & 36.8 & 34.4 & 31.9 & 28.1 & $<0.001$ \\
\hline Fasting blood glucose $100-126$ mg/dl, n & 5.3 & 5.1 & 4.8 & 3.6 & 0.046 \\
\hline Blood pressure $\geq 130 / 85$ mmHg & 44.9 & 45.6 & 42.1 & 42.9 & 0.021 \\
\hline Triglycerides $\geq 150 \mathrm{mg} / \mathrm{dl}$ & 18.7 & 16.9 & 15.6 & 14.2 & $<0.001$ \\
\hline Low HDL-C ${ }^{c}$ & 27.9 & 26.4 & 23.4 & 20.5 & $<0.001$ \\
\hline HOMA-IR & $2.1(1.70)$ & $2.0(1.6)$ & $1.9(1.4)$ & $1.8(1.3)$ & $<0.001$ \\
\hline Hs-CRP, geometric mean (standard error) & $0.14(0.0)$ & $0.14(0.0)$ & $0.12(0.0)$ & $0.12(0.0)$ & $<0.001$ \\
\hline
\end{tabular}

Continuous variables are expressed as mean (standard deviation)

$B M I$ body mass index, $k c a l$ kilocalories, $C V D$ cardiovascular disease, $\mathrm{Hs}$-CRP highly sensitive C-reactive protein, HOMA-IR Homeostatic Model Assessment for Insulin Resistance, $m$ meters, MET metabolic equivalent of task, $p$ points

* Continuous variables were compared across categories of MEDLIFE using anova; categorical variables were compared using chi-squared tests

a At least one self-reported physician-diagnosed disease (coronary heart disease, stroke and heart failure, osteoarthritis, rheumatoid arthritis, respiratory disease, hip fracture, urinary infection, sleep apnea, depression, Alzheimer's disease, cirrhosis, Parkinson's disease, stomach ulcers, intestinal polyps, cataracts, periodontal disease, cancer)

b Abdominal obesity: waist circumference $\geq 102 \mathrm{~cm}$ in men, and $\geq 88$ in women

c $\mathrm{HDL}-\mathrm{C}$ level $<40 \mathrm{mg} / \mathrm{dl}$ in men or $<50$ in women

specifically, the OR for MS was 0.88 (95\% CI 0.83-0.95) while the HR for all-cause mortality was $0.87(0.78$,
0.97) (Additional file 1: Table S4). Within the components of the Block 3, physical activity and collective 
Table 2 Association between quartiles of MEDLIFE index and metabolic syndrome and its components, HOMAR-IR and C-reactive protein in the ENRICA cohort $(\mathrm{N}=11,090)$

\begin{tabular}{|c|c|c|c|c|c|}
\hline & Q1 (5-11 p) & Q2 (12-13 p) & Q3 (14-15 p) & Q4 (16-23 p) & P-trend \\
\hline Total number of participants & 3042 & 3435 & 2917 & 1696 & \\
\hline Metabolic syndrome, cases & 512 & 530 & 385 & 185 & \\
\hline Model 1, OR $(95 \% \mathrm{Cl})$ & 1 Ref. & $0.91(0.77,1.07)$ & $0.80(0.67,0.95)$ & $0.66(0.53,0.83)$ & $<0.001$ \\
\hline Model 2, OR (95\% Cl) & 1 Ref. & $0.88(0.73,1.04)$ & $0.86(0.70,1.04)$ & $0.71(0.56,0.90)$ & 0.007 \\
\hline Model 3, OR (95\% Cl) & 1 Ref. & $0.89(0.74,1.07)$ & $0.87(0.72,1.06)$ & $0.73(0.58,0.93)$ & 0.016 \\
\hline \multicolumn{6}{|l|}{ Components of the metabolic syndrome } \\
\hline Abdominal obesity ${ }^{\mathrm{a}}$, cases & 1111 & 1176 & 927 & 476 & \\
\hline Model 1, OR (95\% Cl) & 1 Ref. & $0.91(0.80,1.04)$ & $0.82(0.71,0.93)$ & $0.68(0.58,0.81)$ & $<0.001$ \\
\hline Model 2, OR (95\% Cl) & 1 Ref. & $0.82(0.69,0.97)$ & $0.83(0.69,0.99)$ & $0.63(0.50,0.79)$ & $<0.001$ \\
\hline Model 3, OR (95\% Cl) & 1 Ref. & $0.82(0.69,0.97)$ & $0.84(0.70,1.00)$ & $0.63(0.51,0.80)$ & $<0.001$ \\
\hline Fasting blood glucose $100-126 \mathrm{mg} / \mathrm{dl}$, cases & 161 & 175 & 139 & 60 & \\
\hline Model 1, OR (95\% CI) & 1 Ref. & $1.07(0.81,1.41)$ & $1.02(0.77,1.36)$ & $0.73(0.51,1.06)$ & 0.193 \\
\hline Model 2, OR (95\% Cl) & 1 Ref. & $1.04(0.78,1.37)$ & $1.04(0.78,1.39)$ & $0.74(0.51,1.07)$ & 0.247 \\
\hline Model 3, OR (95\% Cl) & 1 Ref. & $1.05(0.79,1.39)$ & $1.05(0.79,1.40)$ & $0.75(0.52,1.08)$ & 0.282 \\
\hline Blood pressure $\geq 130 / 85 \mathrm{mmHg}$, cases & 1354 & 1555 & 1217 & 723 & \\
\hline Model 1, OR (95\% Cl) & 1 Ref. & $1.07(0.94,1.22)$ & $0.87(0.75,1.00)$ & $0.90(0.76,1.06)$ & 0.022 \\
\hline Model 2, OR (95\% Cl) & 1 Ref. & $1.08(0.94,1.25)$ & $0.88(0.76,1.01)$ & $0.91(0.77,1.08)$ & 0.046 \\
\hline Model 3, OR (95\% Cl) & 1 Ref. & $1.08(0.94,1.24)$ & $0.92(0.80,1.05)$ & $0.94(0.80,1.10)$ & 0.13 \\
\hline Triglycerides $\geq 150 \mathrm{mg} / \mathrm{dl}$, cases & 568 & 581 & 455 & 240 & \\
\hline Model 1, OR (95\% Cl) & 1 Ref. & $0.89(0.77,1.04)$ & $0.80(0.67,0.94)$ & $0.74(0.61,0.90)$ & 0.001 \\
\hline Model 2, OR (95\% Cl) & 1 Ref. & $0.91(0.78,1.06)$ & $0.87(0.73,1.03)$ & $0.81(0.66,0.99)$ & 0.032 \\
\hline Model 3, OR (95\% Cl) & 1 Ref. & $0.92(0.78,1.07)$ & $0.88(0.74,1.05)$ & $0.83(0.68,1.02)$ & 0.053 \\
\hline Low HDL-C ${ }^{b}$, cases & 847 & 905 & 682 & 348 & \\
\hline Model 1, OR (95\% Cl) & 1 Ref. & $0.87(0.76,0.99)$ & $0.80(0.70,0.92)$ & $0.68(0.58,0.80)$ & $<0.001$ \\
\hline Model 2, OR (95\% Cl) & 1 Ref. & $0.89(0.77,1.03)$ & $0.86(0.74,0.99)$ & $0.75(0.63,0.89)$ & 0.001 \\
\hline Model 3, OR (95\% Cl) & 1 Ref. & $0.89(0.78,1.03)$ & $0.86(0.74,0.99)$ & $0.76(0.63,0.90)$ & 0.001 \\
\hline \multicolumn{6}{|l|}{ HOMA-IR, $95 \% \mathrm{Cl}$, difference of means } \\
\hline Model $1, \beta(95 \% \mathrm{Cl})$ & 0 Ref. & $-0.04(-0.12,0.06)$ & $-0.11(-0.20,-0.02)$ & $-0.21(-0.31,-0.11)$ & $<0.001$ \\
\hline Model 2, $\beta(95 \% \mathrm{Cl})$ & 0 Ref. & $-0.03(-0.12,0.05)$ & $-0.08(-0.16,-0.02)$ & $-0.18(-0.27,-0.09)$ & $<0.001$ \\
\hline Model 3, $\beta(95 \% \mathrm{Cl})$ & 0 Ref. & $-0.03(-0.12,0.05)$ & $-0.06(-0.15,0.02)$ & $-0.17(-0.26,-0.08)$ & 0.001 \\
\hline \multicolumn{6}{|l|}{ Hs-CRP $(\mathrm{mg} / \mathrm{dl})^{c}$, difference of log-means } \\
\hline Model 1, $\beta(95 \% \mathrm{Cl})$ & 0 Ref. & $0.01(-006,0.08)$ & $-0.17(-0.25,-0.10)$ & $-0.13(-0.22,-0.04)$ & $<0.001$ \\
\hline Model 2, $\beta(95 \% \mathrm{Cl})$ & 0 Ref. & $0.02(-0.05,0.09)$ & $-0.14(-0.21,-0.06)$ & $-0.09(-0.17,0.006)$ & $<0.001$ \\
\hline Model 3, $\beta(95 \% \mathrm{Cl})$ & o Ref. & $0.03(-0.05,0.09)$ & $-0.14(-0.21,-0.06)$ & $-0.09(-0.17,0.02)$ & 0.001 \\
\hline
\end{tabular}

Number of morbidities includes the following self-reported physician-diagnosed diseases (coronary heart disease, stroke and heart failure, osteoarthritis, rheumatoid arthritis, respiratory disease, hip fracture, urinary infection, sleep apnea, depression, Alzheimer's disease, cirrhosis, Parkinson's disease, stomach ulcers, intestinal polyps, cataracts, periodontal disease, cancer)

Model 1: adjusted for sex, age, educational level (no formal or primary education, secondary education, university)

Model 2: Adjusted for Model $1+$ smoking (never, former, current), total energy intake (kcal/day), and body mass index

Model 3: Model $2+$ prevalence of cancer, respiratory disease, depression, number of morbidities (excluding the previously mentioned conditions) and number of drug treatments

HDL-C high density lipoprotein cholesterol, Hs-CRP highly sensitive C-reactive protein, HOMA-IR Homeostatic Model Assessment for Insulin Resistance, MEDLIFE Mediterranean lifestyle

a Abdominal obesity: waist circumference $\geq 102 \mathrm{~cm}$ in men, and $\geq 88$ in women

${ }^{b} \mathrm{HDL}-\mathrm{C}$ level $<40 \mathrm{mg} / \mathrm{dl}$ in men or $<50$ in women

c Values are presented for the logarithmic of the hs-CRP 
Table 3 Association between quartiles of the MEDLIFE index and risk of all-cause and cardiovascular death over 8.7 years of follow-up

\begin{tabular}{|c|c|c|c|c|c|c|c|}
\hline & \multicolumn{5}{|c|}{ Categories of adherence to MEDLIFE } & \multirow[t]{2}{*}{$p$ for trend } & \multirow[t]{2}{*}{ Per +2 -point increment } \\
\hline & Q1 (4-11 p) & Q2 (12-13 p) & & Q3 (14-15 p) & Q4 (16-23 p) & & \\
\hline \multicolumn{8}{|c|}{ All-cause mortality } \\
\hline $\mathrm{N}$ & 3042 & 3435 & & 2917 & 1696 & & \\
\hline Deaths & 118 & 106 & & 76 & 30 & & \\
\hline Person-years & $9,716,596$ & $10,978,581$ & & $9,323,908$ & $5,381,351$ & & \\
\hline $\begin{array}{l}\text { Model 1, HR } \\
(95 \% \mathrm{Cl})\end{array}$ & 1 Ref. & $0.92(0.70,1.21)$ & & $\begin{array}{l}0.68(0.48 \\
0.95)\end{array}$ & $\begin{array}{l}0.54(0.36 \\
0.83)\end{array}$ & 0.004 & $0.87(0.79,0.96)$ \\
\hline $\begin{array}{l}\text { Model 2, HR } \\
(95 \% \mathrm{Cl})\end{array}$ & 1 Ref. & $0.91(0.69,1.22)$ & & $\begin{array}{c}0.70(0.49 \\
0.99)\end{array}$ & $\begin{array}{c}0.55(0.36 \\
0.85)\end{array}$ & 0.010 & $0.88(0.80,0.97)$ \\
\hline $\begin{array}{l}\text { Model 3, HR } \\
(95 \% \mathrm{Cl})\end{array}$ & 1 Ref. & $0.88(0.65,1.18)$ & & $\begin{array}{l}0.68(0.48 \\
0.96)\end{array}$ & $\begin{array}{l}0.55(0.35 \\
0.85)\end{array}$ & 0.014 & $0.88(0.79,0.97)$ \\
\hline $\begin{array}{l}\text { Model 4, HR } \\
(95 \% \mathrm{Cl})\end{array}$ & 1 Ref. & $0.92(0.68,1.25)$ & & $\begin{array}{l}0.72(0.51 \\
1.02)\end{array}$ & $\begin{array}{l}0.58(0.37 \\
0.90)\end{array}$ & 0.025 & $0.89(0.81,0.99)$ \\
\hline \multicolumn{8}{|l|}{ CVD mortality } \\
\hline Deaths & 29 & & 21 & 19 & 5 & & \\
\hline Person-years & $9,716,596$ & & $10,978,581$ & $9,323,908$ & $5,381,351$ & & \\
\hline $\begin{array}{l}\text { Model 1, HR } \\
(95 \% \mathrm{Cl})\end{array}$ & 1 Ref. & & $\begin{array}{l}0.50(0.27 \\
0.93)\end{array}$ & $\begin{array}{c}0.72(0.37 \\
1.37)\end{array}$ & $\begin{array}{l}0.26(0.08 \\
0.79)\end{array}$ & 0.02 & $0.78(0.64,0.97)$ \\
\hline $\begin{array}{l}\text { Model 2, HR } \\
\quad(95 \% \mathrm{Cl})\end{array}$ & 1 Ref. & & $\begin{array}{l}0.55(0.29 \\
1.04)\end{array}$ & $\begin{array}{l}0.82(0.42 \\
1.58)\end{array}$ & $\begin{array}{l}0.30(0.10 \\
0.94)\end{array}$ & 0.06 & $0.82(0.67,1.01)$ \\
\hline $\begin{array}{l}\text { Model 3, HR } \\
\quad(95 \% \mathrm{Cl})\end{array}$ & 1 Ref. & & $\begin{array}{l}0.55(0.28 \\
1.07)\end{array}$ & $\begin{array}{c}0.83(0.43 \\
1.62)\end{array}$ & $\begin{array}{l}0.31(0.10 \\
0.94)\end{array}$ & 0.08 & $0.83(0.68,1.02)$ \\
\hline $\begin{array}{c}\text { Model 4, HR } \\
(95 \% \mathrm{Cl})\end{array}$ & 1 Ref. & & $\begin{array}{c}0.57(0.29 \\
1.14)\end{array}$ & $\begin{array}{c}0.87(0.44 \\
1.70)\end{array}$ & $\begin{array}{c}0.33(0.11 \\
1.02)\end{array}$ & 0.11 & $0.84(0.68,1.04)$ \\
\hline
\end{tabular}

The ENRICA cohort, June 2008 to December 2017

Number of morbidities includes the following self-reported physician-diagnosed diseases (coronary heart disease, stroke and heart failure, osteoarthritis, rheumatoid arthritis, respiratory disease, hip fracture, urinary infection, sleep apnea, depression, Alzheimer's disease, cirrhosis, Parkinson's disease, stomach ulcers, intestinal polysp, cataracts, periodontal disease, cancer)

Model 1: adjusted for sex, age, educational level (no formal or primary education, secondary education, university)

Model 2: adjusted for Model $1+$ smoking (never, former, current), total energy intake (Kcal/day), and BMI

Model 3: Model 2 + prevalence of cancer, respiratory disease, depression, number of morbidities (excluding the previously mentioned conditions), number of drug treatments

Model 4: Model 3 + other clinical risk factors, including abdominal obesity (waist circumference $\geq 102 \mathrm{~cm}$ in men, or $\geq 88 \mathrm{~cm}$ in women), fasting blood glucose (100-126 mg/dl and not treated with antidiabetic drugs), high blood pressure (systolic $\geq 130 \mathrm{mmHg}$ or diastolic $\geq 85 \mathrm{mmHg}$ ) or receiving antihypertensive drugs, triglycerides $\geq 150 \mathrm{mg} / \mathrm{dl}$ and serum high-density lipoprotein (HDL) cholesterol $<40 \mathrm{mg} / \mathrm{dl}$ in men or $<50 \mathrm{mg} / \mathrm{dl}$ in women)

$H R$ hazard ratio, $\mathrm{Cl}$ confidence interval

and non-collective sports were driving the association (Additional file 1: Table S5).

\section{Discussion}

\section{Main findings}

In this cohort, representative of the adult Spanish population, a higher adherence to a Mediterranean lifestyle was associated with lower prevalence of the MS and several biological CVD risk factors as well as lower allcause and CVD mortality after an average follow-up of almost 9 years. Indeed, we found that higher adherence to the MEDLIFE index was associated with a relatively $45 \%$ lower all-cause mortality and 69\% relatively lower CVD mortality, but the independent association between
MEDLIFE (as continuous) and CVD mortality was absent after adjustment of multiple covariates, maybe due to low number of cases and thus limit of statistical power Physical activity and conviviality was the only block independently associated with the outcomes of the study. Of note, the MEDLIFE index which represents the Mediterranean lifestyle includes food consumption as well as other dietary habits and health behaviors (conviviality, eating in company, rest and social habits) characteristic of the traditional Mediterranean culture and support the importance of cultural habits, beyond mere food habits, as strong determinants of health. This is important because, despite the evidence of the benefits of Mediterranean diet on multiple outcomes $[14,19,43-45]$, the fact that our 

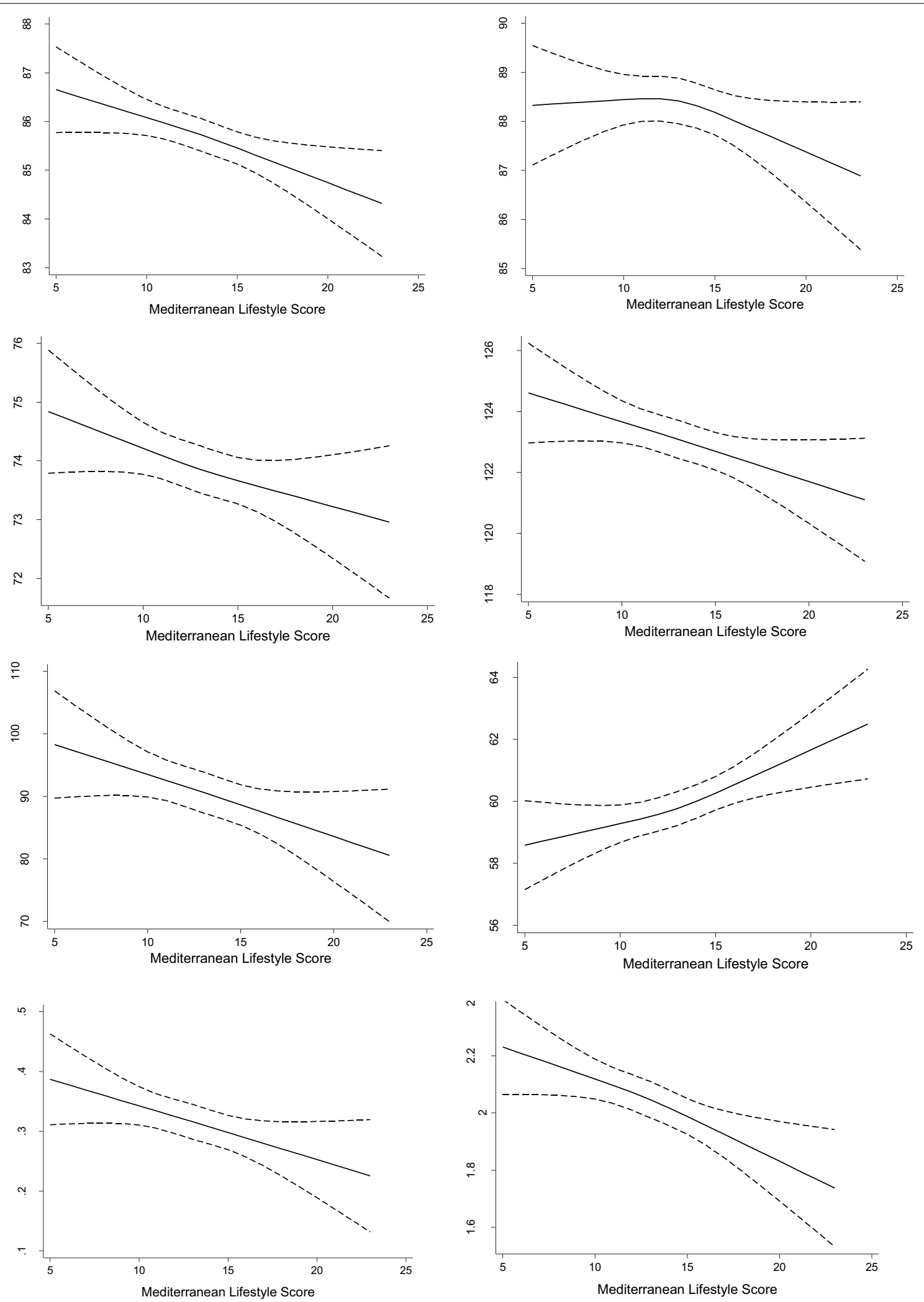

Fig. 1 Dose-response association between MEDLIFE, the components of the metabolic syndrome and hs-CRP, HOMA-IR in ENRICA cohort. Analyses were adjusted as in Model 3. HOMA-IR Homeostatic Model Assessment for Insulin Resistance, Hs-CRP highly sensitive C-reactive protein 


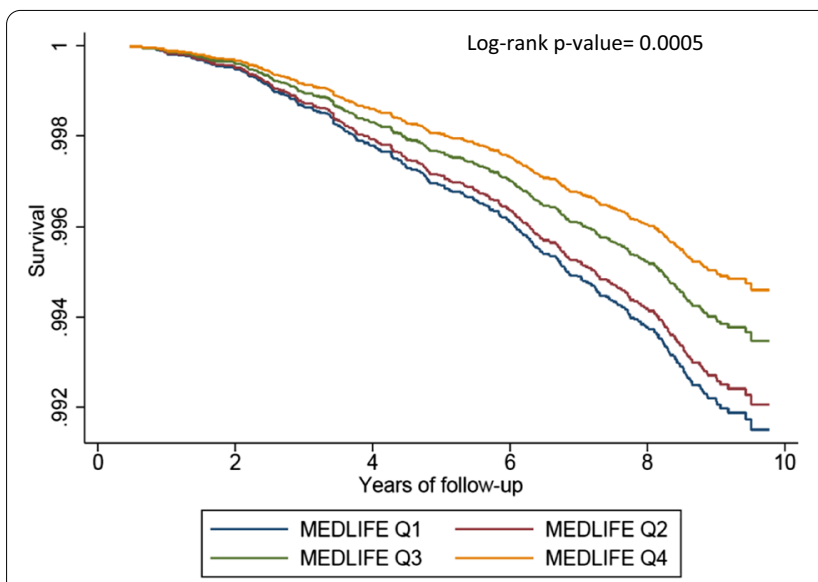

a All-cause mortality

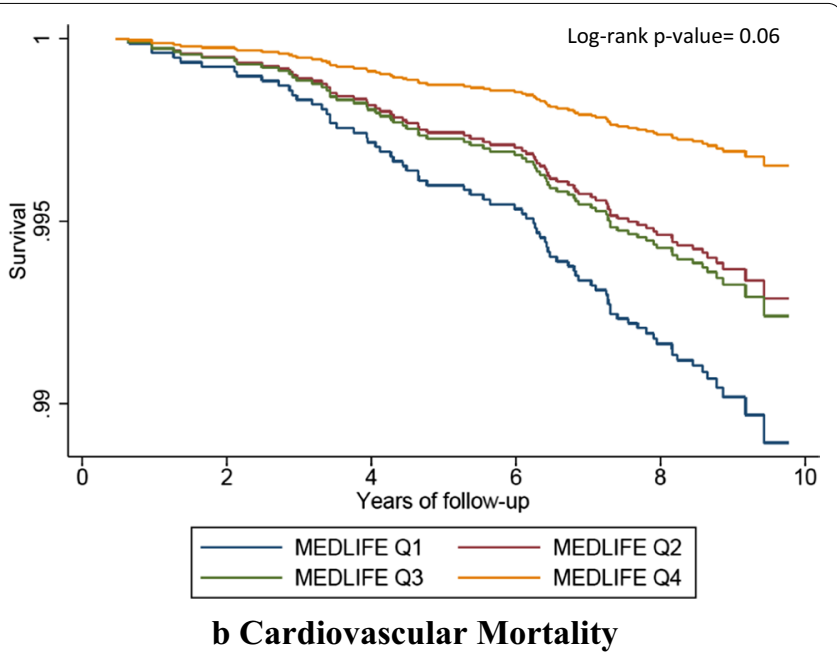

b Cardiovascular Mortality

Fig. 2 Multivariable-adjusted Kaplan-Meier graph for a all cause-death, and b cardiovascular death. Adjusted as in Model 4 in Table 3

study combines a Mediterranean lifestyle all-inclusive is a clear addition to the literature that emphasizes the synergistic effect of the whole lifestyle rather than their individual components. This message can now be addressed to the population within behavioral counseling, and can possibly translate into improved health outcomes.

Our results add to the evidence that a healthy lifestyle is associated with cardiovascular health and inversely associated with all-cause mortality [21, 22, 26, 46-50]. In the large SUN cohort, comprising university graduates in Spain, a better adherence to a joint healthy lifestyle was associated with lower risk of hypertension [49], MS [51], and CVD [23]. Unlike other scores [22, 23, 25, 26, $48,49]$, the MEDLIFE index does not include smoking or BMI because these items (or its absence) do not reflect the Mediterranean lifestyle. Our stratified analyses were robust and the associations were in line with the main analyses by subgroups (by morbidity status, sex, age, age, and BMI) and no significant interaction was found.

\section{Medlife components and the importance of joint effects}

When we assessed the separate effect of the three different blocks of the MEDLIFE, the dimension that captured physical activity, social interaction, rest and conviviality was the only one showing statistically significant results, mainly due to physical activity, collective and non-collective sports. Despite the wide supporting evidence of the benefits of physical activity on cardiovascular health and mortality [52-60], we cannot rule out positive effects of other components such as napping, social interaction, or eating in company that are not usually included in other previously published scores [22, 25, 61]. Our findings may highlight the importance of those components in the context of a healthy Mediterranean dietary pattern. However, we did not assess the isolated effect of each item of the score because we relied on the joint, and possibly synergistic effect of the combination of several behaviors related to the traditional Mediterranean culture; this may contribute to explain why we did not find an association with the other two blocks (capturing food consumption and dietary components). Indeed, lifestyle behaviors are often correlated, thus by simultaneously examining the effect of several lifestyle variables, we accounted for the clustering of healthful types of behavior within the same individual. Additionally, the MEDLIFE score was not designed with the aim of assessing each block separately but to evaluate the comprehensive Mediterranean lifestyle reflecting culture and tradition. Therefore, the main drivers of the association could be different in each population but the overall effect may provide a better understanding of the whole Mediterranean lifestyle, tradition and culture in a holistic approach, and therefore adding the novelty of going beyond the exclusive focus on the foods consumed.

\section{Lifestyle scores and cardiovascular health}

Our results are in line with those of previous studies showing the benefits of a healthy lifestyle on the MS. In a long-term observational study of women, more than one-half of CVD risk factor diagnoses (hypertension, diabetes, high cholesterol) could have been prevented if all of them had optimal levels of 6 lifestyle factors (not smoking, normal BMI, physical activity $\geq 2.5 \mathrm{~h} /$ week, television $\leq 7 \mathrm{~h} /$ week, diet in top $40 \%$ of the Alternative Healthy Eating Index-2010, and 0.1-14.9 g/day of alcohol) [22]. Also a systematic review of 11 interventions found that lifestyle modifications on MS were effective in 
resolving the proportions of patients with MS in comparison with conventional education [62].

Several studies evaluating other lifestyle scores like the American Heart Association Life Simple 7, that includes 4 lifestyle behaviors (BMI, smoking, diet, physical activity) and 3 clinical risk factors (blood pressure, high cholesterol, and diabetes) have found important inverse associations with CVD and total-mortality in different cohorts [63-68]. In addition, reductions in alcohol intake and energy intake over 1 year in participants with diabetes diagnosis was association with lower hazard of CVD [69]. However, the effect of behavioral factors may be attenuated after the development of the clinical risk factors which are associated with CVD. In our study, after adjustment for the clinical risk factors, the association between MEDLIFE and risk of CVD and all-cause death only slightly changed. While this suggests that the expected protective association of the Mediterranean lifestyle on mortality is substantially greater than that expected only from the reduced frequency of the MS and other clinical risk factors also observed in our study, no etiological conclusion can be drawn.

\section{Strengths and limitations}

Strengths of our study include the long follow-up and large size of the cohort, which is representative of the adult population of a whole country and allows for generalization of the results. Lifestyle information included very detailed questions with a validated dietary history comprising a variety of foods and cooking methods, and a validated physical activity questionnaire. In addition, we used a previously validated lifestyle index (MEDLIFE) reflecting the Mediterranean culture and lifestyle that includes common components across the Mediterranean countries reflecting culture and tradition (social interaction, short nap, meals in company, physical activity with others, etc.). Limitations of this study include potential measurement errors due to the self-reported nature of some of the data, although this bias is most likely to be non-differential and thus would drive the association towards the null. Lifestyle information was only assessed at baseline and, thus, no changes over time were assumed, which could dilute the actual associations. In addition, despite extensive adjustment for known risk factors, some residual confounding may persist; to address this issue we calculated the E-value [42], and it showed that after accounting for confounders measured in this study, the HR of 0.58 for total mortality could only be explained away by an unmeasured confounder that was associated with both the MEDLIFE and mortality with a risk ratio of more than 3.26 (upper CI, 1.12). Thus, given the high E-value (3.26), it seems unlikely that an unmeasured confounder could fully explain away the association between the MEDLIFE and mortality. Additionally, although our results come from a representative Spanish population, generalizability to non-Mediterranean countries or different ethnic groups should be explored. Lastly, although the MEDLIFE aims to be a comprehensive Mediterranean lifestyle index, it is possible that factors not considered, such as spending time in open-air spaces [70], belonging to extended families, or having religious beliefs and practices (mostly Catholic) in Southern Europe may also contribute to cardiovascular health $[71,72]$.

\section{Conclusion}

In conclusion, a higher adherence to a MEDLIFE index was associated with lower frequency of the MS and reduced all-cause mortality in Spain. Future studies should determine if this also applies to other Mediterranean countries, and whether a Mediterranean-like lifestyle may improve cardiovascular health outside the Mediterranean basin.

\section{Supplementary Information}

The online version contains supplementary material available at https://doi. org/10.1186/s12933-020-01195-1.

Additional file 1: Table S1. Description of the Mediterranean Lifestyle (MEDLIFE) index modified for ENRICA. Table S1. Description of the Mediterranean Lifestyle (MEDLIFE) index modified for ENRICA. Table S2. Subgroup analyses for the association between MEDLIFE quartiles and Metabolic Syndrome. Table S3. Subgroup analyses for the association between MEDLIFE quartiles and risk of all-cause death. Table S4. Association between each main block of the MEDLIFE index and the metabolic syndrome and all-cause mortality. Table S5. Association between each component of Block 3 (Physical Activity and Conviviality) and the metabolic syndrome and all-cause mortality. Figure S1. Flowchart of eligible participants in the ENRICA cohort. Figure S2. Restricted cubic spline for each 1-point increment of MEDLIFE and risk of all-cause mortality (A) and Cardiovascular Disease (CVD) mortality (B).

\section{Abbreviations}

MS: Metabolic syndrome; CVD: Cardiovascular disease; MedDiet: Mediterranean diet; BMI: Body mass index; MEDLIFE: Mediterranean lifestyle index; HR: Hazard ratio; OR: Odds ratio; Cl: Confidence interval; HDL-c: High-density lipoprotein cholesterol; HOMA-IR: Homoeostatic Model Assessment for Insulin Resistance Index; hs-CRP: High-sensitivity C-reactive protein.

\section{Acknowledgements}

Not applicable.

\section{Authors' contributions}

MSP formulated the study question and design, performed the statistical analyses, interpreted the results, and drafted the manuscript. RO, contributed to the statistical modeling. MSP, EGE, EL, RO, and FRA contributed to the conception and design of the study and acquisition of the data. All authors contributed to the interpretation of data and critical revision of the manuscript. MSP and FRA share primary responsibility for the final content. All authors read and approved the final manuscript.

\section{Funding}

This work was supported by FIS grants 16/609, 16/1512, 18/287, 19/319 and PI20/00896 (Instituto de Salud Carlos III, State Secretary of R+D+I and FEDER/ FSE), the Salamander Project (JPI-A Healthy Diet for a Healthy Life, State Secretary of R+D+I PCIN-2016-145), and the Cátedra de Epidemiología y Control 
del Riesgo Cardiovascular at UAM (\#820024). Mercedes Sotos Prieto holds a Ramón y Cajal contract (RYC-2018-025069-I) from the Ministry of Science, Innovation and Universities and FEDER/FSE. The funding agencies had no role in study design, data collection and analysis, interpretation of results, manuscript preparation or in the decision to submit this manuscript for publication.

\section{Availability of data and materials \\ Not applicable.}

\section{Ethics approval and consent to participate}

Study participants gave written informed consent, and the study was approved by the Clinical Research Ethics Committee of La Paz University Hospital in Madrid (Trial registration: NCT02804672).

\section{Consent for publication}

Not applicable.

\section{Competing interests}

The authors declare that they have no competing interests.

\section{Author details}

1 Department of Preventive Medicine and Public Health, School of Medicine, Universidad Autónoma de Madrid, IdiPaz (Instituto de Investigación Sanitaria Hospital Universitario La Paz), and CIBERESP (CIBER of Epidemiology and Public Health), Madrid, Spain. ${ }^{2}$ Department of Environmental Health, Harvard T.H. Chan School of Public Health, Boston, MA, USA. ${ }^{3}$ Department of Preventive Medicine and Public Health, University of Navarra, IdiSNA, Pamplona, Spain. ${ }^{4}$ Biomedical Research Network Centre for Pathophysiology of Obesity and Nutrition (CIBEROBN), Carlos III Health Institute, Madrid, Spain. ${ }^{5}$ Department of Nutrition, Harvard T.H. Chan School of Public Health, Boston, MA, USA. ${ }^{6}$ IMDEA-Food Institute, CEI UAM+CSIC, Madrid, Spain.

Received: 25 September 2020 Accepted: 9 December 2020 Published online: 05 January 2021

\section{References}

1. Hidalgo-Mora JJ, Garcia-Vigara A, Sanchez-Sanchez ML, Garcia-Perez MA, Tarin J, Cano A. The Mediterranean diet: a historical perspective on food for health. Maturitas. 2020;132:65-9.

2. Bach-Faig A, Berry EM, Lairon D, Reguant J, Trichopoulou A, Dernini S, et al. Mediterranean diet pyramid today. Science and cultural updates. Public Health Nutr. 2011;14:2274-84.

3. Kastorini C-M, Milionis HJ, Esposito K, Giugliano D, Goudevenos JA, Panagiotakos DB. The effect of Mediterranean diet on metabolic syndrome and its components: a meta-analysis of 50 studies and 534,906 individuals. J Am Coll Cardiol. 2011;57:1299-313.

4. Esposito K, Kastorini C-M, Panagiotakos DB, Giugliano D. Mediterranean diet and metabolic syndrome: an updated systematic review. Rev Endocr Metab Disord. 2013;14:255-63.

5. Godos J, Zappala G, Bernardini S, Giambini I, Bes-Rastrollo M, MartinezGonzalez M. Adherence to the Mediterranean diet is inversely associated with metabolic syndrome occurrence: a meta-analysis of observational studies. Int J Food Sci Nutr. 2017;68:138-48.

6. Consortium TI. Mediterranean diet and type 2 diabetes risk in the European prospective investigation into cancer and nutrition (EPIC) study: the InterAct project. Diabetes Care. 2011;34:1913-8.

7. Esposito K, Maiorino Ml, Bellastella G, Chiodini P, Panagiotakos D, Giugliano D. A journey into a Mediterranean diet and type 2 diabetes: a systematic review with meta-analyses. BMJ Open. 2015;5:e008222.

8. Schwingshackl L, Missbach B, Konig J, Hoffmann G. Adherence to a Mediterranean diet and risk of diabetes: a systematic review and metaanalysis. Public Health Nutr. 2015;18:1292-9.

9. Jannasch F, Kröger J, Schulze MB. Dietary patterns and type 2 diabetes: a systematic literature review and meta-analysis of prospective studies. J Nutr. 2017;147:1174-82

10. Esposito K, Kastorini CM, Panagiotakos DB, Giugliano D. Mediterranean diet and weight loss: meta-analysis of randomized controlled trials. Metab Syndr Relat Disord. 2011;9:1-12.
11. Echeverría G, McGee EE, Urquiaga I, Jiménez P, D'Acuña S, Villarroel L, et al. Inverse associations between a locally validated Mediterranean diet index, overweight/obesity, and metabolic syndrome in Chilean adults. Nutrients. 2017;9:862.

12. Sotos-Prieto M, Bhupathiraju SN, Mattei J, Fung TT, Li Y, Pan A, et al. Changes in diet quality scores and risk of cardiovascular disease among US men and women. Circulation. 2015;132:2212-9.

13. Sotos-Prieto M, Bhupathiraju SN, Mattei J, Fung TT, Li Y, Pan A, et al. Association of changes in diet quality with total and cause-specific mortality. N Engl J Med. 2017;377:143-53.

14. Estruch R, Ros E, Salas-Salvadó J, Covas M-I, Corella D, Arós F, et al. Primary prevention of cardiovascular disease with a Mediterranean diet supplemented with extra-virgin olive oil or nuts. N Engl J Med. 2018;378:e34.

15. Martínez-González MA, Gea A, Ruiz-Canela M. The Mediterranean diet and cardiovascular health. Circ Res. 2019;124:779-98.

16. Corina A, Abrudan MB, Nikolic D, Cătoi AF, Chianetta R, Castellino G, et al. Effects of aging and diet on cardioprotection and cardiometabolic risk markers. Curr Pharm Des. 2019;25:3704-14.

17. Schwingshackl L, Schwedhelm C, Galbete C, Hoffmann G. Adherence to Mediterranean diet and risk of cancer: an updated systematic review and meta-analysis. Nutrients. 2017;9:1063.

18. Fung TT, Rexrode KM, Mantzoros CS, Manson JE, Willett WC, Hu FB. Mediterranean diet and incidence of and mortality from coronary heart disease and stroke in women. Circulation. 2009;119:1093-100.

19. Rosato V, Temple NJ, La Vecchia C, Castellan G, Tavani A, Guercio V. Mediterranean diet and cardiovascular disease: a systematic review and meta-analysis of observational studies. Eur J Nutr. 2017;58(1):173-91.

20. Martínez-González MA, Guillén-Grima F, De Irala J, Ruíz-Canela M, BesRastrollo M, Beunza JJ, et al. The Mediterranean diet is associated with a reduction in premature mortality among middle-aged adults. J Nutr. 2012;142:1672-8.

21. Sotos-Prieto M, Bhupathiraju SN, Falcon LM, Gao X, Tucker KL, Mattei J. A healthy lifestyle score is associated with cardiometabolic and neuroendocrine risk factors among Puerto Rican adults. J Nutr. 2015;145:1531-40.

22. Chomistek AK, Chiuve SE, Eliassen AH, Mukamal KJ, Willett WC, Rimm EB. Healthy lifestyle in the primordial prevention of cardiovascular disease among young women. J Am Coll Cardiol. 2015;65:43-51.

23. Diaz-Gutierrez J, Ruiz-Canela M, Gea A, Fernandez-Montero A, MartinezGonzalez MA. Association between a healthy lifestyle score and the risk of cardiovascular disease in the SUN cohort. Rev Esp Cardiol. 2018;71:1001-9.

24. Li Y, Pan A, Wang DD, Liu X, Dhana K, Franco OH, et al. Impact of healthy lifestyle factors on life expectancies in the US population. Circulation. 2018;138(4):345-55.

25. Li Y, Schoufour J, Wang DD, Dhana K, Pan A, Liu X, et al. Healthy lifestyle and life expectancy free of cancer, cardiovascular disease, and type 2 diabetes: prospective cohort study. BMJ. 2020;368:16669.

26. van den Brandt PA. The impact of a Mediterranean diet and healthy lifestyle on premature mortality in men and women. Am J Clin Nutr. 2011;94:913-20.

27. Nyberg ST, Singh-Manoux A, Pentti J, Madsen IEH, Sabia S, Alfredsson $L$, et al. Association of healthy lifestyle with years lived without major chronic diseases. JAMA Intern Med. 2020;180(5):760-8.

28. Sotos-Prieto M, Moreno-Franco B, Ordovas JM, Leon M, Casasnovas JA, Penalvo JL. Design and development of an instrument to measure overall lifestyle habits for epidemiological research: the Mediterranean lifestyle (MEDLIFE) index. Public Health Nutr. 2015;18:959-67.

29. Sotos-Prieto M, Santos-Beneit G, Bodega P, Pocock S, Mattei J, Penalvo JL. Validation of a questionnaire to measure overall Mediterranean lifestyle habits for research application: the Mediterranean lifestyle index (Medlife). Nutr Hosp. 2015;32:1153-63.

30. Pavičić Žeželj S, Kenđel Jovanović G, Krešić G. The association between the Mediterranean diet and high physical activity among the working population in Croatia. Med Pr. 2019;70:169-76.

31. Rodriguez-Artalejo F, Graciani A, Guallar-Castillon P, Leon-Munoz LM, Zuluaga MC, Lopez-Garcia E, et al. Rationale and methods of the study on nutrition and cardiovascular risk in Spain (ENRICA). Rev Esp Cardiol. 2011;64:876-82.

32. Guallar-Castillon P, Sagardui-Villamor J, Balboa-Castillo T, Sala-Vila A, Ariza Astolf MJ, Sarrion Pelous MD, et al. Validity and reproducibility of a Spanish dietary history. PLoS ONE. 2014;9:e86074. 
33. Wareham NJ, Jakes RW, Rennie KL, Schuit J, Mitchell J, Hennings S, et al. Validity and repeatability of a simple index derived from the short physical activity questionnaire used in the European prospective investigation into cancer and nutrition (EPIC) study. Public Health Nutr. 2003;6:407-13.

34. Martinez-Gonzalez MA, Lopez-Fontana C, Varo JJ, Sanchez-Villegas A, Martinez JA. Validation of the Spanish version of the physical activity questionnaire used in the nurses' health study and the health professionals' follow-up study. Public Health Nutr. 2005;8:920-7.

35. Alberti KGMM, Eckel RH, Grundy SM, Zimmet PZ, Cleeman II, Donato KA, et al. Harmonizing the metabolic syndrome: a joint interim statement of the International Diabetes Federation Task Force on Epidemiology and Prevention; National Heart, Lung, and Blood Institute; American Heart Association; World Heart Federation; International Atherosclerosis Society; and International Association for the Study of Obesity. Circulation. 2009;120:1640-5.

36. Guallar-Castillón P, Pérez RF, López García E, León-Muñoz LM, Aguilera MT, Graciani A, et al. Magnitude and management of metabolic syndrome in Spain in 2008-2010: the ENRICA study. Rev Esp Cardiol (Engl Ed). 2014;67:367-73.

37. Matthews DR, Hosker JP, Rudenski AS, Naylor BA, Treacher DF, Turner RC. Homeostasis model assessment: insulin resistance and beta-cell function from fasting plasma glucose and insulin concentrations in man. Diabetologia. 1985;28:412-9.

38. Informes Metodológicos Estandarizados. https://www.ine.es/dynt3/metad atos/es/RespuestaPrint.html?oper=23. Accessed 10 Nov 2020.

39. Cirera L, Salmerón D, Martínez C, Bañón RM, Navarro C. More than a decade improving medical and judicial certification in mortality statistics of death causes. Rev Esp Salud Publica. 2018;92:e201806031.

40. Cirera SL. First biennium of mortality statistics with, the automatic system Iris for coding multiple causes of death. Gac Sanit. 2018;32:5-7.

41. Groenwold RHH, Klungel OH, Grobbee DE, Hoes AW. Selection of confounding variables should not be based on observed associations with exposure. Eur J Epidemiol. 2011;26:589-93.

42. VanderWeele TJ, Ding P. Sensitivity analysis in observational research: introducing the E-value. Ann Intern Med. 2017;167:268-74.

43. Bouzas C, Bibiloni MDM, Julibert A, Ruiz-Canela M, Salas-Salvadó J, Corella D, et al. Adherence to the Mediterranean lifestyle and desired body weight loss in a mediterranean adult population with overweight: a PREDIMED-plus study. Nutrients. 2020;12:2114.

44. Bautista MC, Engler MM. The Mediterranean diet: is it cardioprotective? Prog Cardiovasc Nurs. 2005:20:70-6.

45. Michaëlsson K, Baron JA, Byberg L, Höijer J, Larsson SC, Svennblad B, et al. Combined associations of body mass index and adherence to a Mediterranean-like diet with all-cause and cardiovascular mortality: a cohort study. PLoS Med. 2020;17:e1003331.

46. Liu G, Li Y, Hu Y, Zong G, Li S, Rimm EB, et al. Influence of lifestyle on incident cardiovascular disease and mortality in patients with diabetes mellitus. J Am Coll Cardiol. 2018;71:2867-76.

47. Behrens G, Fischer B, Kohler S, Park Y, Hollenbeck AR, Leitzmann MF. Healthy lifestyle behaviors and decreased risk of mortality in a large prospective study of US women and men. Eur J Epidemiol. 2013;28:361-72.

48. Van Dam RM, LiT, Spiegelman D, Franco OH, Hu FB. Combined impact of lifestyle factors on mortality: prospective cohort study in US women. BMJ. 2008:337:a1440-a1440.

49. Diaz-Gutierrez J, Ruiz-Estigarribia L, Bes-Rastrollo M, Ruiz-Canela M, MartinMoreno JM, Martinez-Gonzalez MA. The role of lifestyle behaviour on the risk of hypertension in the SUN cohort: the hypertension preventive score. Prev Med. 2019;123:171-8.

50. Zhu N, Yu C, Guo Y, Bian Z, Han Y, Yang L, et al. Adherence to a healthy lifestyle and all-cause and cause-specific mortality in Chinese adults: a 10-year prospective study of 0.5 million people. Int J Behav Nutr Phys Act. 2019;16:98.

51. Garralda-Del-Villar M, Carlos-Chilleron S, Diaz-Gutierrez J, Ruiz-Canela M, Gea A, Martinez-Gonzalez MA, et al. Healthy lifestyle and incidence of metabolic syndrome in the SUN cohort. Nutrients. 2018;11:65.

52. Mok A, Khaw K-T, Luben R, Wareham N, Brage S. Physical activity trajectories and mortality: population based cohort study. BMJ. 2019:365:12323.

53. Kraus WE, Powell KE, Haskell WL, Janz KF, Campbell WW, Jakicic JM, et al. Physical activity, all-cause and cardiovascular mortality, and cardiovascular disease. Med Sci Sports Exerc. 2019;51:1270-81.

54. Martinez-Gomez D, Lavie CJ, Hamer M, Cabanas-Sanchez V, Garcia-Esquinas E, Pareja-Galeano H, et al. Physical activity without weight loss reduces the development of cardiovascular disease risk factors - a prospective cohort study of more than one hundred thousand adults. Prog Cardiovasc Dis. 2019:62:522-30.

55. Martinez-Gomez D, Ortega FB, Hamer M, Lopez-Garcia E, Struijk E, Sadarangani KP, et al. Physical activity and risk of metabolic phenotypes of obesity: a prospective Taiwanese cohort study in more than 200,000 adults. Mayo Clin Proc. 2019:94:2209-19.

56. Pedersen LR, Olsen RH, Anholm C, Astrup A, Eugen-Olsen J, Fenger M, et al. Effects of 1 year of exercise training versus combined exercise training and weight loss on body composition, low-grade inflammation and lipids in overweight patients with coronary artery disease: a randomized trial. Cardiovasc Diabetol. 2019;18:127.

57. Medina C, Coxson P, Penko J, Janssen I, Bautista-Arredondo S, Barquera S, et al. Cardiovascular and diabetes burden attributable to physical inactivity in Mexico. Cardiovasc Diabetol. 2020;19:99.

58. Schröder H, Cárdenas-Fuentes G, Martínez-González MA, Corella D, Vioque J, Romaguera D, et al. Effectiveness of the physical activity intervention program in the PREDIMED-Plus study: a randomized controlled trial. Int J Behav Nutr Phys Act. 2018;15:110.

59. Eser P, Marcin T, Prescott E, Prins LF, Kolkman E, Bruins W, et al. Clinical outcomes after cardiac rehabilitation in elderly patients with and without diabetes mellitus: the EU-CaRE multicenter cohort study. Cardiovasc Diabetol. 2020;19:37.

60. Nesti L, Pugliese NR, Sciuto P, Natali A. Type 2 diabetes and reduced exercise tolerance: a review of the literature through an integrated physiology approach. Cardiovasc Diabetol. 2020;19:134

61. Sotos-Prieto M, Mattei J, Hu FB, Chomistek AK, Rimm EB, Willett WC, et al. Association between a healthy heart score and the development of clinical cardiovascular risk factors among women: potential role for primordial prevention. Circ Cardiovasc Qual Outcomes. 2016;9:S77-85.

62. Yamaoka $K$, Tango T. Effects of lifestyle modification on metabolic syndrome: a systematic review and meta-analysis. BMC Med. 2012;10:138.

63. Dong C, RundekT, Wright CB, Anwar Z, Elkind MS, Sacco RL. Ideal cardiovascular health predicts lower risks of myocardial infarction, stroke, and vascular death across whites, blacks, and hispanics: the northern Manhattan study. Circulation. 2012;125:2975-84

64. Dong Y, Hao G, Wang Z, Wang X, Chen Z, Zhang L. Ideal cardiovascular health status and risk of cardiovascular disease or all-cause mortality in Chinese middle-aged population. Angiology. 2019;70:523-9.

65. Fang $N$, Jiang $M$, Fan $Y$. Ideal cardiovascular health metrics and risk of cardiovascular disease or mortality: a meta-analysis. Int J Cardiol. 2016;214:279-83.

66. Guo L, Zhang S. Association between ideal cardiovascular health metrics and risk of cardiovascular events or mortality: a meta-analysis of prospective studies. Clin Cardiol. 2017;40:1339-46.

67. Hwang SJ, Onuma O, Massaro JM, Zhang X, Fu YP, Hoffmann U, et al. Maintenance of ideal cardiovascular health and coronary artery calcium progression in low-risk men and women in the Framingham heart study. Circ Cardiovasc Imaging. 2018;11:e006209.

68. Díez-Espino J, Buil-Cosiales P, Babio N, Toledo E, Corella D, Ros E, et al. Impact of life's simple 7 on the incidence of major cardiovascular events in high-risk Spanish adults in the PREDIMED study cohort. Rev Esp Cardiol (Engl Ed). 2020;73:205-11.

69. Strelitz J, Ahern AL, Long GH, Boothby CE, Wareham NJ, Griffin SJ. Changes in behaviors after diagnosis of type 2 diabetes and 10-year incidence of cardiovascular disease and mortality. Cardiovasc Diabetol. 2019;18:98.

70. Manferdelli G, La Torre A, Codella R. Outdoor physical activity bears multiple benefits to health and society. J Sports Med Phys Fitness. 2019;59:868-79.

71. ShattuckEC, Muehlenbein MP. Religiosity/spirituality and physiological markers of health. J Relig Health. 2020;59:1035-54.

72. Kim ES, VanderWeele TJ. Mediators of the association between religious service attendance and mortality. Am J Epidemiol. 2019;188:96-101.

\section{Publisher's Note}

Springer Nature remains neutral with regard to jurisdictional claims in published maps and institutional affiliations. 